SANDIA REPORT

Re Apperal Desk for

SAND98-1813

Unlimited Release

Printed August 1998

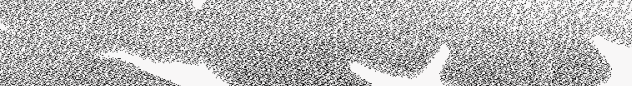

A Finite Element-Boundary Element

Method for Advection-Diffusion Problems

with Variable Advective Fields and Infinite

Domains

Brian J. Driessen, Jeffrey L. Dohner

Prepared by

Sandia National Laboratories

Albuquerque, New Mexico 87185 and Livermore, California 94550

Sandia is a multiprogram laboratory operated by Sandia Corporation, a Lockheed Martin Company, for the United States Department of

Energy under Contract DE-AC04-94AL85000.

Approved for public release; further dissemination unlimited.
PEC

SEP 1988

OSTI 
Issued by Sandia National Laboratories, operated for the United States Department of Energy by Sandia Corporation.

NOTICE: This report was prepared as an account of work sponsored by an agency of the United States Government. Neither the United States Government nor any agency thereof, nor any of their employees, nor any of their contractors, subcontractors, or their employees, makes any warranty, express or implied, or assumes any legal liability or responsibility for the accuracy, completeness, or usefulness of any information, apparatus, product, or process disclosed, or represents that its use would not infringe privately owned rights. Reference herein to any specific commercial product, process, or service by trade name, trademark, manufacturer, or otherwise, does not necessarily constitute or imply its endorsement, recommendation, or favoring by the United States Government, any agency thereof, or any of their contractors or subcontractors. The views and opinions expressed herein do not necessarily state or reflect those of the United States Government, any agency thereof, or any of their contractors.

Printed in the United States of America. This report has been reproduced directly from the best available copy.

Available to DOE and DOE contractors from

Office of Scientific and Technical Information

P.O. Box 62

Oak Ridge, TN 37831

Prices available from (615) 576-8401, FTS 626-8401

Available to the public from

National Technical Information Service

U.S. Department of Commerce

5285 Port Royal Rd

Springfield, VA 22161

NTIS price codes

Printed copy: A03

Microfiche copy: A01

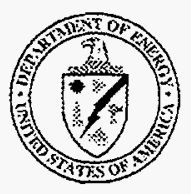




\section{DISCLAIMER}

Portions of this document may be illegible in electronic image products. Images are produced from the best available original document. 
SAND98-1813

Unlimited Release

Printed August 1998

\title{
A FINITE ELEMENT-BOUNDARY ELEMENT METHOD FOR ADVECTION-DIFFUSION PROBLEMS WITH VARIABLE ADVECTIVE FIELDS AND INFINITE DOMAINS
}

\author{
Brian J. Driessen \\ Jeffrey L. Dohner \\ Structural Dynamics Department \\ Sandia National Laboratories \\ P.O. Box 5800 \\ Albuquerque, NM 87185-0439
}

\begin{abstract}
In this paper a hybrid, finite element - boundary element method which can be used to solve for particle advection-diffusion in infinite domains with variable advective fields is presented. In previous work either boundary element, finite element, or difference methods have been used to solve for particle motion in advective-diffusive domains. These methods have a number of limitations. Due to the complexity of computing spatially dependent Green's functions, the boundary element method is limited to domains containing only constant advective fields, and due to their inherent formulation, finite element and finite difference methods are limited to only domains of finite spatial extent. Thus, finite element and finite difference methods are limited to finite space problems for which the boundary element method is not, and the boundary element method is limited to constant advection field problems for which finite element and finite difference methods are not. In this paper it is proposed to split a domain into two sub-domains, and for each of these sub domains, apply the appropriate solution method; thereby, producing a method for the total infinite space, variable advective field domain.
\end{abstract}





\section{Acknowledgment}

Special thanks goes to Dr. Rush Robinett and Dr. Barry Spletzer for funding this research. And additional thanks also goes to Dr. Walter Gerstle for his insights, and Julie Kesti and Theresa Garley and Glenda Sweatt for their library assistance. 



\section{Contents}

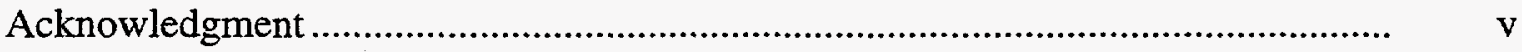

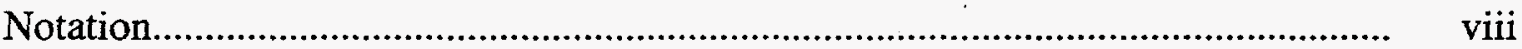

\section{A FINITE ELEMENT-BOUNDARY ELEMENT METHOD FOR ADVECTION-DIFFUSION PROBLEMS WITH VARIABLE ADVECTIVE FIELDS AND INFINITE DOMAINS}

1 Introduction ................................................................................................

2 Derivation of Equations of Motion ...................................................................... 3

3 Discretization of the Equation of Motion ………...................................................

3.1 A FEM Approximation of the Equation of Motion ............................................ 6

3.2 A BEM Approximation of the Equation of Motion ............................................. 8

3.3 Coupling of the FEM and BEM Equations ..................................................... 10

4 Numerical Results................................................................................. 11

4.1 Numerical Solution for a Constant Advective Field......................................... 11

4.2 Numerical Solution for a Variable Advective Field ……................................... 13

4.3 Numerical Solution for Realistic Obstacles ...................................................... 15

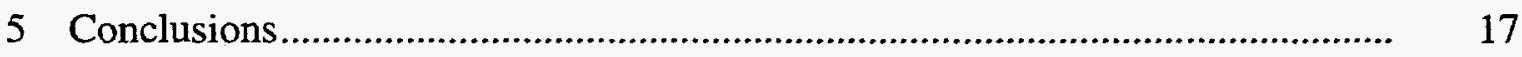

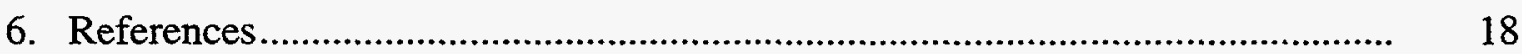

\section{Figures}

Fig. 1 Mass conservation of species $i$ through the control volume $\Omega$...................... 3

Fig. 2 A schematic of a hybrid FEM/BEM domain .................................................

Fig. 3 Problem 1 geometry: Particles diffuse from a point source in a domain with a constant advective field .....................................................................

Fig. 4 Problem 1 results: A comparison of the FEM/BEM solution to the exact solution, o - FEM/BEM solution, -- exact solution ..................................... 12

Fig. 5 Problem 2 geometry: Particles diffuse from a spherical source in a domain with a non constant advective field

Fig. 6 Problem 2 results: A plot of particle concentration at locations in the $x y$ plane for a 2750 and 3250 DOF mesh, o- 2750 DOF mesh, +-3250 DOF mesh

Fig. 7 Problem 3 geometry: Two buildings surrounded by an infinite half space .....

Fig. 8 Problem 3 results: Particles diffuse in a variable advective field around buildings. 


\section{Notation}

$\phi_{i}$ - concentration of a species $i$

$\vec{V}$ - mass-averaged velocity of the

medium

$\alpha$ - diffusivity of the medium

$\rho$ - average density of the medium

$\rho_{i}$ - density of species $i$ in the medium

$\vec{V}_{i}$-particle velocity of the species $i$

$\Gamma$ - surface of control volume

$\vec{n}$ - normal vector

$W$ - bases function

$\Omega_{F E M}$ - interior domain where FEM is applicable

$\Omega_{B E M}$ - exterior domain where BEM is applicable

$\Gamma_{\text {in }}$ - surface of obstacles

$\Gamma_{\text {out }}$ - surface shared by $\Omega_{F E M}$ and

$\Omega_{B E M}$

$N_{i n}$ - number of nodes on $\Gamma_{i n}$

$N_{\text {out }}$ - number of nodes on $\Gamma_{\text {out }}$

$\Phi-\left(\phi_{i_{1}}, \ldots \phi_{i_{N}}\right)^{T}$ $\phi_{i_{j}}-j^{\text {th }} \phi_{i}$ value at nodes in $\Omega_{F E M}$

$\Phi_{i n}-$ vector of $\phi_{i}$ values on $\Gamma_{i n}$

$\Phi_{\text {out }}$ - vector of $\phi_{i}$ values on $\Gamma_{\text {out }}$

$\frac{\partial \Phi_{i n}}{\partial n}$ - vector of normal derivatives of

$\phi_{i}$ on $\Gamma_{\text {in }}$

$\frac{\partial \Phi_{o u t}}{\partial n}$ - vector of normal derivatives of

$\phi_{i}$ on $\Gamma_{\text {out }}$

$A, B, C$ - FEM matrices

$G$ - Green's function

$\vec{r}_{0}$ and $\vec{r}$ - points in $\Omega_{B E M}$

$M, D, G$ - BEM matrices

$\psi$ - velocity potential

$u \vec{\imath}$ - velocity of wind at infinity 


\section{INTRODUCTION}

Numerical methods are used to analyze the advection and diffusion of particles in complex domains. Although a number of numerical methods for advection-diffusion analysis presently exist, most are applicable to problems with domains of infinite spatial extent and constant advective fields or to problems with domains of finite spatial extent and variable advective fields, but few are applicable to problems with domain of both infinite spatial extent and variable advective fields. In this paper, a method will be presented which can be used to solve for a sub set of advective-diffusion problems with infinite spatial domains and variable advective fields.

Although much has been written on the numerical solution of advection-diffusion problems, the infinite space problem with non constant advective fields is still immature. Qiu et al [1] used a Boundary Element Method (BEM) for solving an infinite space advectiondiffusion problem with very high Peclet number. However, in their analysis, they used the Green's function associated with a constant advective field; therefore, their analysis was only valid for problems with constant field characteristics. Similar in form to advectiondiffusion, convection-diffusion problems have been studied extensively in the thermal sciences. Li and Evans [2] used an exponential variable transformation to construct a variational principle which lead to a symmetric banded finite element stiffness matrix. As with Qiu et al, they assumed the convective field was constant; therefore their solution is limited. Moreover, since they use a Finite Element Method (FEM), they were limited to finite spatial domains. Taigbenu and Liggett [3] used the non convective Green's function in an integral approach to model convective domains. This required a domain integration which when discretized leads to fully dense large domain matrices. Their method could model 
convection-diffusion with non constant convective fields; however, it was valid only for domains of finite spatial extent.

Liggett [4] gives a very good discussion of the applicability of the BEM and the extent to which it can be used for advection-diffusion problems. The main points discussed included the fact that the BEM, when it can be applied, is much easier to use than either finite differences or finite elements. The method is inexpensive in terms of human effort (set-up time) and computer run-time. Another main point was that the BEM can handle free surfaces more easily than domain methods; however, it was noted that finite element and finite difference methods can be applied to a larger set of applications.

In conclusion, while problems with either finite domains with variable advective fields or infinite domains with constant advective fields have been studied extensively, problems with infinite space domains and variable advective fields have been relatively untouched. In the following sections, we present a method which allows for the modeling of particle motion in infinite space domains with variable advective fields produced by complex obstacle boundaries. In this presentation, it is assumed that the total domain can be partitioned into two sub domains: one sub domain is infinite and contains a constant advective field and the other sub domain is finite and contains a variable advective field. The sub domain with the variable advective fields is modeled using the FEM, and the sub domain with constant advective fields is modeled using the BEM. 


\section{DERIVATION OF EQUATION OF MOTION}

In this section we derive the differential equation of motion for particle advection and diffusion in an incompressible medium. This derivation is obtained by using Fick's Law of diffusion and conservation of mass. In later sections, this equation of motion will be approximated using a FEM and BEM.

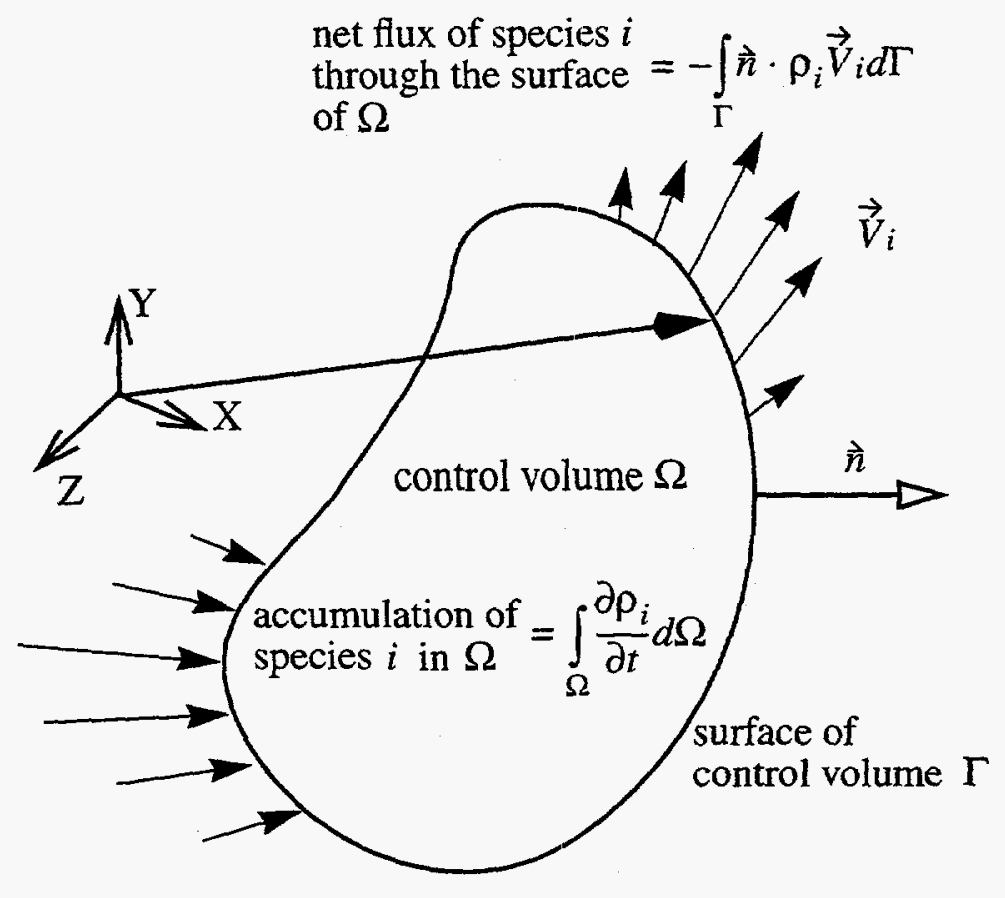

Figure 1. Mass conservation of species $i$ through the control volume $\Omega$

Consider the control volume in Figure 1. A fluid medium carries a distribution of diffusive particle species though this volume. Let $\phi_{i}$ be the concentration of a species $i$ in the medium, $\vec{V}$ be the mass-averaged velocity of the medium, $\alpha$ be the diffusivity of the medium, $\rho$ be the average density of the medium, and $\rho_{i}$ be the density of the species $i$ in the medium. From Fick's Law of diffusion, the particle velocity of the species $i, \vec{V}_{i}$, is the 
sum of two components--the advection component, $\vec{V}$, and the diffusion component, $-\frac{\rho \alpha}{\rho_{i}} \nabla \phi_{i}[5]$. This can be stated analytically as

$$
\vec{V}_{i}=\vec{V}-\frac{\rho}{\rho_{i}} \alpha \nabla \phi_{i}
$$

Notice that if the species concentration, $\phi_{i}$, is uniform, then the species simply moves at the mass averaged velocity, $\vec{V}$. On the other hand, if $\phi_{i}$ is not uniform, then the species has a velocity relative to $\vec{V}$ where the relative velocity, $\vec{V}_{i}-\vec{V}$, is in the "downhill" direction of the concentration field.

A differential advection-diffusion equation of motion for $\phi_{i}$ can be determine by using (1) and by imposing mass conservation. Assuming no internal particle production, from the conservation of mass,

$$
\begin{aligned}
& \text { accumulation of } \\
& \text { species } i \text { in } \Omega
\end{aligned}=\begin{aligned}
& \text { net flux of species } i \text { through } \\
& \text { the surface of } \Omega .
\end{aligned}
$$

In analytical terms, the accumulation of species $i$ in $\Omega$ is

$$
\int_{\Omega}^{\partial \rho_{i}} \frac{\partial}{\partial t} d \Omega
$$

where $\rho_{i}=\phi_{i} \rho$. Moreover, letting $\Gamma$ be the surface of $\Omega$ and $\vec{n}$ the outward unit normal vector, then the net flux though $\Gamma$ is given by

$$
-\int_{\Gamma} \vec{n} \cdot \rho_{i} \vec{V}_{i} d \Gamma=-\int_{\Omega} \nabla \cdot\left(\rho_{i} \vec{V}_{i}\right) d \Omega
$$

Combining (4) and (1), and noting that $\nabla \cdot \vec{V}=0$ for an incompressible medium,

$$
-\int_{\Gamma} \vec{n} \cdot \rho_{i} \vec{V}_{i} d \Gamma=-\int_{\Omega}\left(\vec{V} \cdot \nabla \rho_{i}-\rho \alpha \nabla^{2} \phi_{i}\right) d \Omega
$$


Combining (2), (3), and (5) gives

$$
\frac{\partial \phi_{i}}{\partial t}=-\vec{V} \cdot \nabla \phi_{i}+\alpha \nabla^{2} \phi_{i}
$$

which is the governing partial differential equation of motion for incompressible advection-diffusion.

Notice that if $\vec{V}$ were a variable, (6) would be a non-linear equation. However, if $\vec{V}$ is a known quantity then (6) reduces to a linear problem for $\phi_{i}$. Therefore, in this paper, to avoid the complexity of non-linear analysis, the solution for $\phi_{i}$ will be decomposed into two steps. In the first step, the mean wind velocity, $\vec{V}$, is calculated assuming potential flow (this does not require any knowledge of $\phi_{i}$ ), and in the second step, the solution $\vec{V}$ is substituted into (6) and $\phi_{i}$ is calculated. Since calculation of the first step is usually straight forward, the rest of this paper will be focused toward the calculation of the second step.

In general, (6) cannot be solved for in closed form; therefore, numerical methods must be used. To solve (6) using a FEM or BEM, it must be placed into a weak formulation. A steady state weak formulation of (6) for a trial function $W$ is

$$
\int_{\Omega} W\left(\alpha \nabla^{2} \phi_{i}-\vec{V} \cdot \nabla \phi_{i}\right) d \Omega=0
$$

In the following section, a FEM and BEM approximation will be formulated using (7). 


\section{DISCRETIZATION OF THE EQUATION OF MOTION}

In this section the equation of motion will be discretized using a FEM and BEM. In many problems, obstacles reside in a bounded, finite domain of limited extent, and at distances removed from these obstacles, the mean velocity, $\vec{V}$, is practically constant. As will be shown, a BEM can be used to model particle motion at locations removed from these obstacles, and a FEM can be used to model particle motion at locations in the vicinity of these obstacles. In the following subsections, a FEM and BEM are used to produce approximations to a weak form of the equation of motion (equation 7). These approximations are valid for limited sub domains. To model the total domain, the two approximations are then coupled at their domain interfaces.

\subsection{A FEM approximation of the equation of motion}

Consider the simple domain shown in Figure 2.

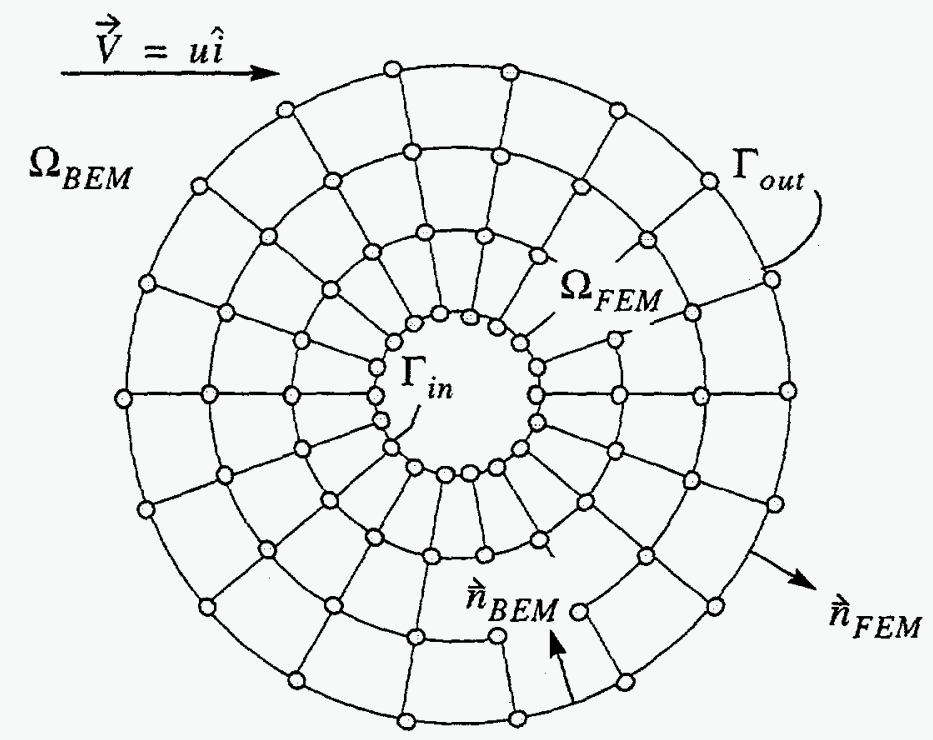

Figure 2. A schematic of a hybrid FEM/BEM domain 
In this domain $\Omega_{F E M}$ is an interior, finite sub domain with a variable advective field that can be modeled by using FEM, and $\Omega_{B E M}$ is an exterior, infinite sub domain with a constant advective field that can be modeled by using BEM. Let $N$ denote the number of nodes in $\Omega_{F E M}, \Phi=\left(\phi_{i_{1}}, \ldots \phi_{i_{N}}\right)^{T}$ be a vector containing the values of $\phi_{i}$ at node locations, $\Gamma_{\text {in }}$ denote the surface of the obstacles in $\Omega_{F E M}$, and $\Gamma_{\text {out }}$ denote the exterior surface that bounds $\Omega_{F E M}$. Let $N_{i n}$ be the number of nodes on the inner surface, $\Gamma_{i n}$, and $N_{\text {out }}$ be the number of nodes on the outer surface, $\Gamma_{\text {out }}$. Let $\Phi_{\text {in }}$ be the vector of nodal values of $\phi_{i}$ on $\Gamma_{i n}, \Phi_{\text {out }}$ be the vector of nodal values of $\phi_{i}$ on $\Gamma_{\text {out }}, \frac{\partial \Phi_{\text {in }}}{\partial n}$ be the vector of normal derivatives of $\phi_{i}$ on $\Gamma_{i n}$, and $\frac{\partial \Phi_{\text {out }}}{\partial n}$ be the vector of normal derivatives of $\phi_{i}$ on $\Gamma_{\text {out }}$.

A relation between $\Phi, \frac{\partial \Phi_{\text {out }}}{\partial n}$, and $\frac{\partial \Phi_{\text {in }}}{\partial n}$ can be obtained by a Galerkin approach. Replacing $W$ in (7) with a finite element basis function $w_{j}$, where $j=1, \ldots, N$, we obtain

$$
\int_{\Omega_{F E M}}\left(\alpha w_{j} \nabla^{2} \phi_{i}-w_{j} \vec{V} \cdot \nabla \phi_{i}\right) d \Omega_{F E M}=0
$$

Applying the first form of Green's theorem to the first term of (8) gives

$$
\begin{aligned}
-\int_{\Omega_{F E M}}\left(\alpha \nabla \phi_{i} \cdot \nabla w_{j}\right) d \Omega_{F E M} & +\int_{\Gamma_{i n}}\left(\alpha w_{j} \frac{\partial \phi_{i}}{\partial n}\right) d \Gamma_{i n}+ \\
& \int_{\Gamma_{\text {out }}}\left(\alpha w_{j} \frac{\partial \phi_{i}}{\partial n}\right) d \Gamma_{i n}-\int_{\Omega_{F E M}}\left(w_{j} \vec{V} \cdot \nabla \phi_{i}\right) d \Omega_{F E M}=0
\end{aligned}
$$


Letting

$$
\phi_{i}=\sum_{j=1}^{N} \phi_{i_{j}} w_{j},
$$

taking the summation outside the integrals and performing the resulting integrations for each $j$, one arrives at a matrix equation of the form

$$
A \Phi+B\left(\frac{\partial \Phi_{o u t}}{\partial n}\right)+C\left(\frac{\partial \Phi_{i n}}{\partial n}\right)=0
$$

Equation 11 is a FEM formulation for modeling steady state advection and diffusion in the bounded domain, $\Omega_{F E M}$. This formulation is not limited to a constant $\vec{V}$ field since $A$ is a function of $\vec{V}$, but is limited to finite space domains and small Peclet numbers. Since high wind velocities are not of concern in this paper, the limitation due to the Peclet number is not of relevance; however, the limitation due to the infinite spatial domain is of relevance and is overcome by coupling this solution to a BEM formulation. In the next subsections, this formulation and its coupling to equation 11 will be discussed.

\subsection{A BEM approximation of the equation of motion}

The steady state equation of motion can also be expressed in integral equation form, and from this form, a BEM can be used to produce a discrete approximation. The integral representation is derived from (7) and the Green's function, G. For constant advective fields, this Green's function can be easily computed; however, for variable advection, calculation of the Green's function becomes complex. Therefore, the BEM is seldom used to model particle motion in non constant advective domains. In this paper, the BEM is used to model particle motion in only the constant advection portion of the total domain. 
Replacing the basis function $W$ in (7) with the Green's function $G$, the weak form becomes

$$
\int_{\Omega_{B E M}}\left(\alpha G \nabla^{2} \phi_{i}-G \vec{V} \cdot \nabla \phi_{i}\right) d \Omega_{B E M}=0
$$

Applying the divergence theorem and the second form of Green's theorem to (12) gives

$$
\int_{\Omega_{B E M}}\left(\alpha \phi_{i} \nabla^{2} G+\phi_{i} \vec{V} \cdot \nabla G\right) d \Omega_{B E M}=\int_{\Gamma_{\text {out }}}\left(\vec{n} \cdot\left(-\alpha G \nabla \phi_{i}+\alpha \phi_{i} \nabla G+\phi_{i} G \vec{V}\right)\right) d \Gamma_{\text {out }} .
$$

Since, by definition of the Green's function, $G$, satisfies

$$
\alpha \nabla^{2} G+\vec{V} \cdot \nabla G=-\delta\left(\vec{r}_{0}-\grave{r}\right)
$$

where $\grave{r}_{0}$ and $\vec{r}$ are points in $\Omega_{B E M}$, (13) becomes

$$
c_{0}\left(\vec{r}_{0}\right) \phi\left(\vec{r}_{0}\right)=\int_{\Gamma_{\text {out }}} \vec{n} \cdot\left(\alpha G \nabla \phi_{i}-\alpha \phi_{i} \nabla G-\phi_{i} G \vec{V}\right) d \Gamma_{\text {out }}
$$

where $c_{0}$ is determined by the surface solid angle at $\vec{r}_{0}$.

When $\vec{V}$ is not a constant or is not a very simple function of spatial location, the closed form solution to (14) is difficult to calculate; however, when $\vec{V}$ is constant, the closed form solution for $G$ is well known (see Qiu et al [1]) and is given by

$$
G\left(\grave{r}, \grave{r}_{0}\right)=\frac{1}{4 \pi \alpha R} e^{\frac{-u}{2 \alpha}\left(R+\left(x-x_{0}\right)\right)}
$$

where

$$
R \equiv\left|\grave{r}_{0}-\vec{p}\right|
$$

$\vec{V}=\vec{u}$, and $u$ is a constant.

Equation 15 is an integral representation of the equation of motion. Since (14) is difficult to solve for when $\vec{V}$ is not a constant, this equation of motion is seldom (if ever) used to model problems with non constant advective fields. Nevertheless, since it contains only a surface integration, it can easily be used to model infinite space problems. 
The surface integral in (15) can be approximated using the BEM. Using shape functions on $\Gamma_{\text {out }}$ that are compatible with the shape functions in (8), one can arrive at a matrix equation of the form

$$
c_{0} \Phi_{\text {out }}=M \Phi_{\text {out }}+G\left(\frac{\partial \Phi_{\text {out }}}{\partial n}\right)
$$

From (18), we have

$$
\Phi_{o u t}=D \Phi=\left(c_{0} \boldsymbol{I}-\mathbf{M}\right)^{-1} G\left(\frac{\partial \Phi_{o u t}}{\partial n}\right)
$$

\subsection{Coupling of the FEM and BEM equations}

The coupled $N+N_{\text {out }}$ equations, (11) and (19), can be solved simultaneously to yield the

variables $\Phi$ and $\frac{\partial \Phi_{\text {out }}}{\partial n}$. In particular with $\left(\frac{\partial \Phi_{\text {in }}}{\partial n}\right)$ known, the coupled matrix equation to be solved is

$$
\left[\begin{array}{cc}
\boldsymbol{A} & \boldsymbol{B} \\
\boldsymbol{D} & -\left(c_{0} \boldsymbol{I}-\boldsymbol{M}\right)^{-1} \boldsymbol{G}
\end{array}\right]\left[\begin{array}{c}
\boldsymbol{\Phi} \\
\left(\frac{\partial \Phi_{o u t}}{\partial n}\right)
\end{array}\right]=\left[\begin{array}{c}
-C\left(\frac{\partial \Phi_{i n}}{\partial n}\right) \\
0
\end{array}\right] .
$$

Equation (20) is mostly sparse with $\mathrm{O}(N)$ non zero entries except for the relatively small dense sub-matrix in the lower right associated with the BEM. It can be solved with an iterative method such as the generalized minimum residual method [6] or with a direct sparse solver. For most problems, solving (20) is not difficult since $N+N_{\text {out }}$ is usually small. 


\section{NUMERICAL RESULTS}

Fluid flow about obstacles produces non constant advective fields; however, in many problems, when no obstacles are present, the advective field is or almost is constant. As described in Section 3, advection-diffusion in finite space domains with non constant advective fields can be modeled using FEM while the advection-diffusion in infinite domains with constant advective fields can be modeled using BEM. Therefore, near obstacles a FEM is used to model particle motion and away from obstacles a BEM method is used. In this section results using this hybrid FEM-BEM of solution are presented. When an exact solution exists, it will be presented with these results for the purpose of quantifying numerical error.

Three problems will be presented in this section. In the first problem, a point source diffuses particles into an infinite domain in the presence of constant wind. A closed form solution exists for this problem; therefore, a comparison between the exact and numerical solutions can be made. In the second problem, the point source is replaced with a source of spherical geometry, and in the third problem, the FEM-BEM is used to model particle motion around a set of realistic complex obstacles.

\subsection{Numerical solution for a constant advective field}

The first problem is shown in Figure 3. A constant flux of particles flow from a point source in an infinite domain. Within the domain a constant wind is blowing. Therefore, the advective field is constant. The solution to this problem is well known [7] and therefore, provides a method to verify the FEM-BEM solution. 


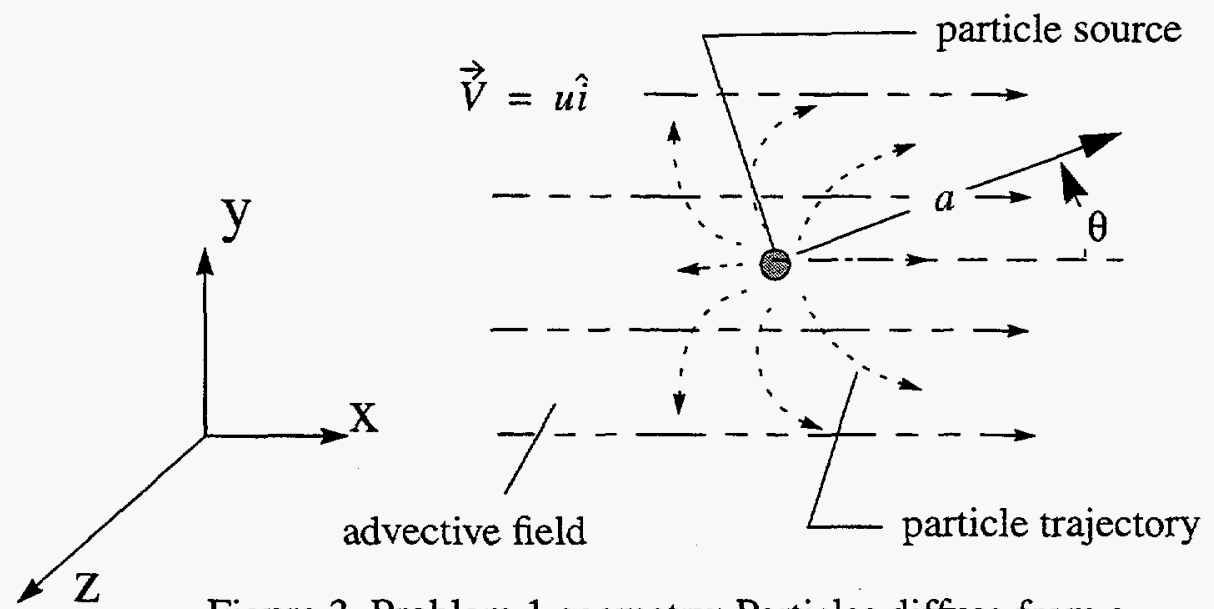

Figure 3. Problem 1 geometry: Particles diffuse from a point source in a domain with a constant advective field

Figure 2 is an illustration of the mesh used to solve this problem. Due to the difficulty of applying a Dirac Delta function to model the point source in the FEM domain, the center portion of the mesh has been removed and the forcing term $\frac{\partial \Phi_{i n}}{\partial n}$ was calculated from the exact solution and applied on $\Gamma_{i n}$. The value of $\phi_{i}$ could then be predicted at points in

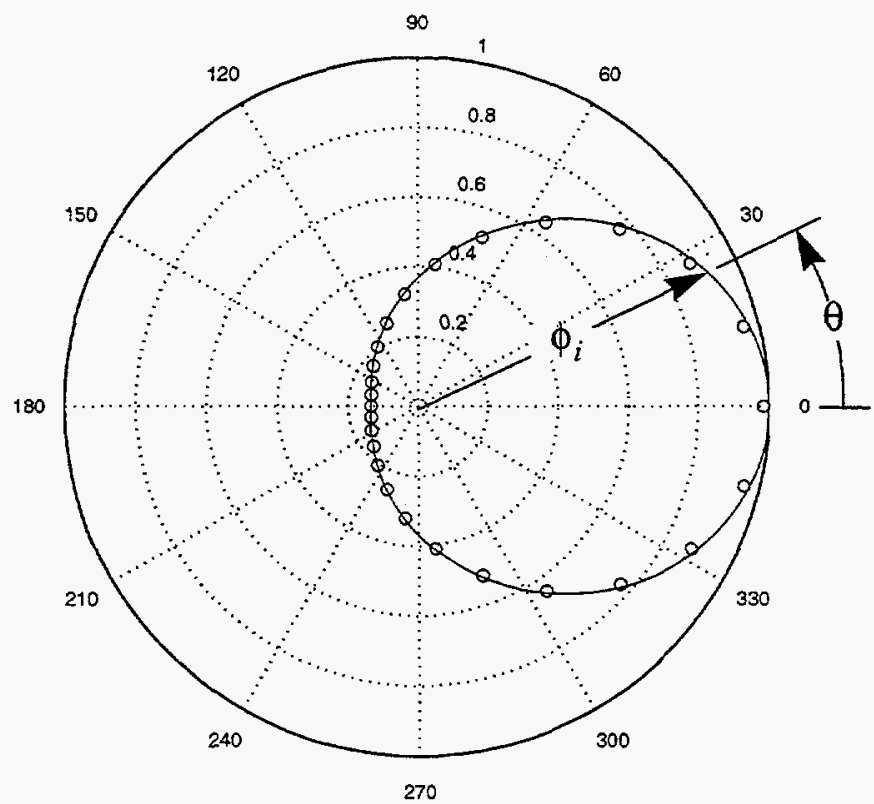

Figure 4. Problem 1 results: A comparison of the FEM/BEM solution to the exact solution, o - FEM/BEM solution, -- exact solution 
$\Omega_{F E M}$ and $\Omega_{B E M}$.

In Figure 4, the closed form and the numerical solution are compared. In this figure, the value of $\phi_{i}$ is plotted for $a=1$ and various values of $\theta$ where $\theta$ is the angle and $a$ is the magnitude of a vector in the xy plane shown in Figure 3. In this example, $a=1$, and the vector points to points in $\Gamma_{i n}$. Figure 4 is a polar plot with radial distance equal to $\phi_{i}$ for various $\theta$ values. The maximum error shown in this plot between the FEM-BEM solution and the exact solution is $2 \%$. Overall the FEM-BEM solution agreed very well with the exact solution.

\subsection{Numerical solution for a variable advective field}

The second problem is shown in Figure 5. In this problem, particles flow from a spherical source. The source not only emits particles but also alters the flow of wind in the domain. Therefore, the advective field is not constant but varies near the source; however, far from the source, the wind flow and therefore the advective field is almost constant.

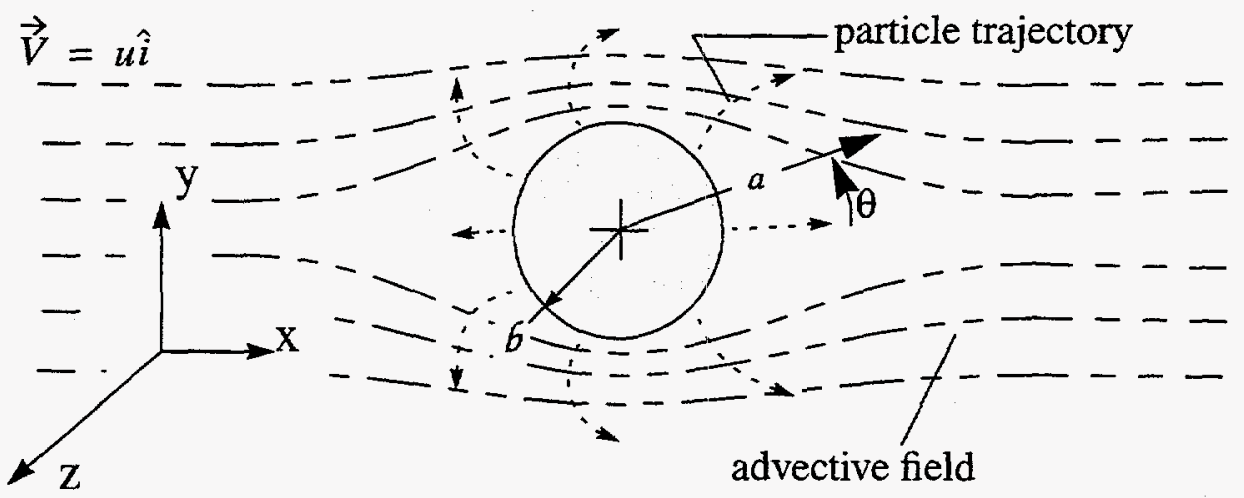

Figure 5. Problem 2 geometry: Particles diffuse from a spherical source in a domain with a non constant advective field 
An exact solution for the flow of wind around a spherical obstacle exists [8]. If $\psi$ is the mean velocity potential, then for this obstacle

$$
\psi=u x+\frac{u b^{3} x}{2 a^{3}}
$$

where $b$ is the radius of the obstacle, $a$ is the distance from the center of the obstacle, $\vec{V}=\nabla \psi$, and $x=a \cdot \cos \theta$ as $\theta$ and $a$ are defined in Figure 5. The difference between $u \vec{\imath}$, the velocity at infinity, and the true velocity at any point in $\Omega_{F E M}$ or $\Omega_{B E M}$ decays as $\frac{b^{3}}{a^{3}}$ where the biggest difference between these velocities occurs along the $\mathrm{x}$ axis. For the true velocity to be within $2 \%$ of $u \vec{i}, a \approx 3 b$. In other words, for this problem, the finite element mesh must be about 2 obstacle radii thick or must have a radius 3 times that of the obstacle for the solution to be accurate.

The mesh used to model this problem is also illustrated in Figure 2. The boundary conditions for a uniform particle flux were applied on $\Gamma_{\text {in }}$, and the resulting coupled equations (20) were used to solve for $\Phi$ and $\frac{\partial \Phi_{\text {out }}}{\partial n}$. A polar plot of $\phi_{i}$ versus $\theta$ on the circle $a=1$ is given in Figure 6 for two different mesh densities. As seen in this figure, for these mesh densities, the solution has converged 


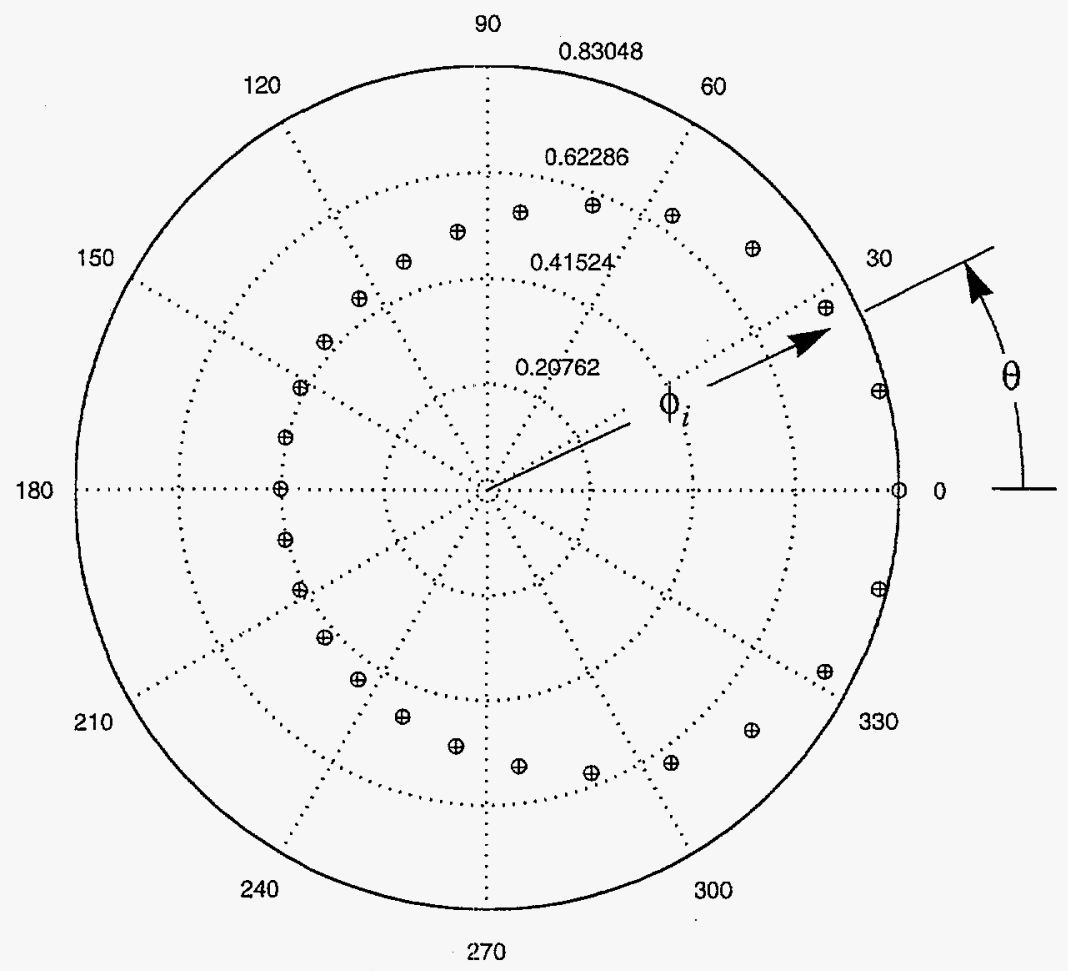

Figure 6. Problem 2 results: A plot of particle concentration at locations in the xy plane for a 2750 and 3250 DOF mesh, o - 2750 DOF mesh, + - 3250 DOF mesh

\subsection{Numerical solution for realistic obstacles}

A more realistic problem is illustrated in Figure 7. A set of buildings block the flow of wind in an infinite space domain. In proximity to these buildings is a particle source distribution. This distribution emits particles into the domain which both diffuse through the wind and are carried by the wind around and over the buildings. The buildings are assumed to be impervious to both the diffusion of the particles and to the flow of the wind. 


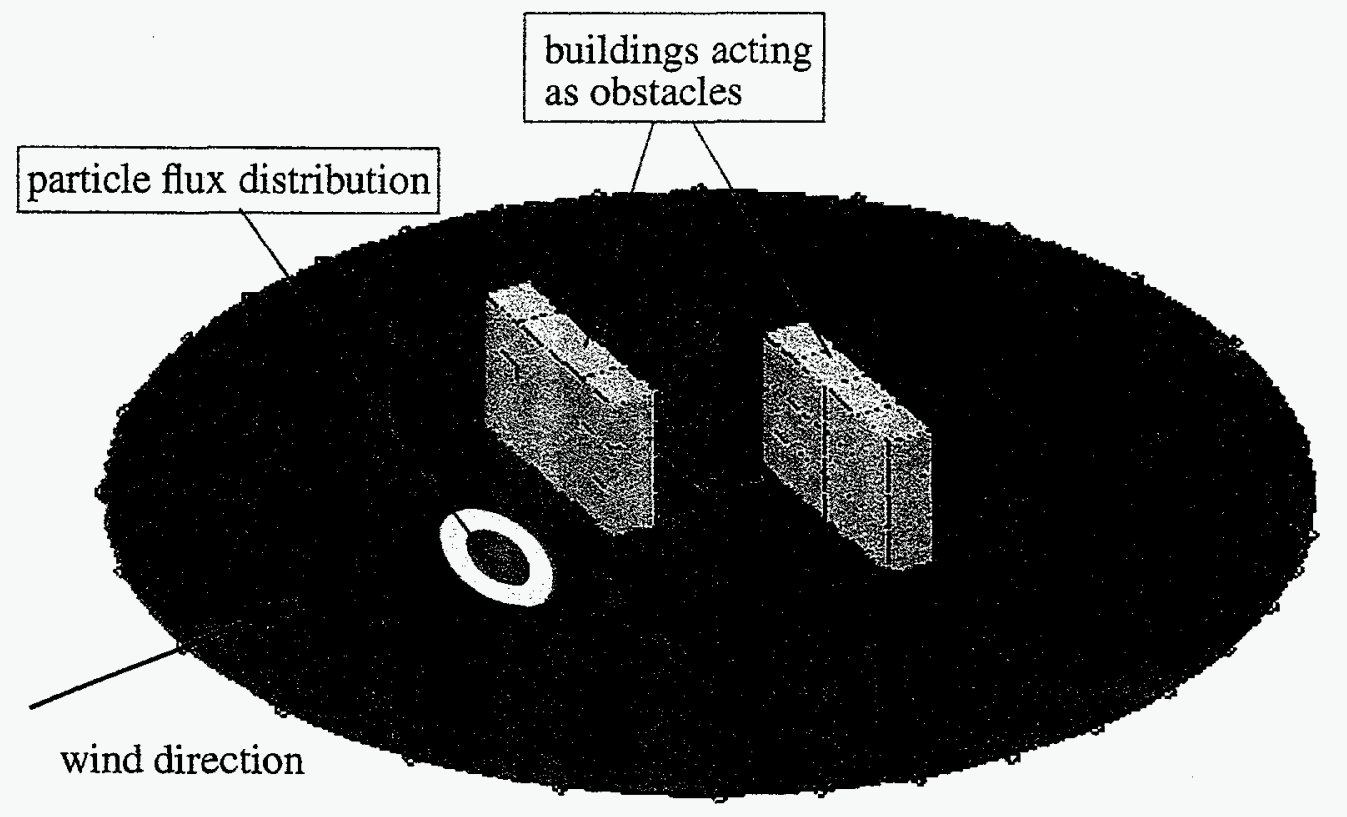

Figure 7. Problem 3 geometry: Two buildings surrounded by an infinite half space

Using the FEM-BEM solution developed in this paper, this complex problem was solved. First the flow field around the buildings was numerically determined using standard potential theory. Then the domain was divided into sub-domains with almost constant and variable advective fields. The BEM method was applied to the constant advective field sub-domain and the FEM was applied to the variable advective field subdomain. The two solutions were then coupled and a total advective-diffusion solution was solved for. A resultant particle concentration plot is shown in Figure 8. 


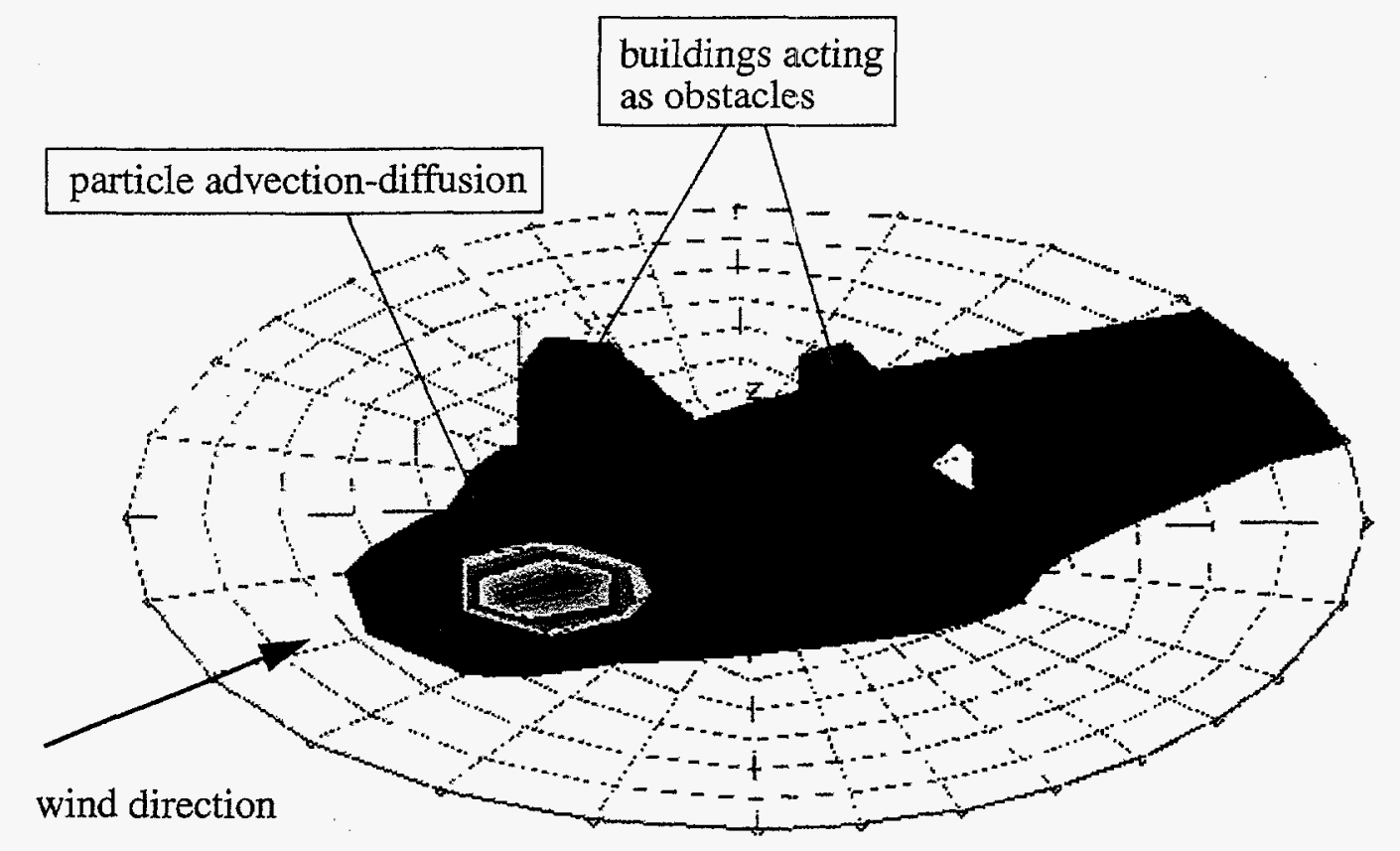

Figure 8. Problem 3 results: Particles diffuse in a variable advective field around buildings.

\section{CONCLUSIONS}

In this paper, a hybrid finite element - boundary element method (FEM-BEM) of solution was presented for a set of advection-diffusion problems. For many problems, the advective field is variable close to obstacles in the domain, but at distances removed from those obstacles, the field is almost constant. By placing finite element meshes around obstacles where the advective field varies and by using the BEM at locations removed from these obstacles, one can solve a set of advective-diffusion problems which are seldom addressed in the present literature. 


\section{REFERENCES}

1. Z.H. Qiu, L.C. Wrobel and H. Power, 'Numerical solution of convection-diffusion problems at high Peclet number using boundary elements', Int. J. Numer. Methods Eng., 41, 899-914, (1998).

2. B.Q. Li and J.W. Evans, 'Boundary element solution of heat convection-diffusion problems', J. Comput. Phys., 93, 255-272, (1991).

3. A. Taigbenu and J.A. Liggett, 'An integral formulation applied to the diffusion and Boussinesq equations', Int. J. Numer. Methods Eng., 23, 1057-1079, (1986).

4. J.A. Liggett, 'Advances in the boundary integral equation method in subsurface flow', Water Resour. Bull., 23(4), 637-651, (1987).

5 D.K. Edwards, V.E. Denny and A.F. Mills, Transfer Processes, 2nd edn, McGraw-Hill Book Company, New York, 1979.

6. Y. Saad and M. Schultz, 'GMRES: A generalized minimum residual algorithm for solving nonsymmetric linear systems', SIAM J. Sci. Stat. 7(3), 856-869, 1986.

7. O.G. Sutton, Micrometeorology, A Study of Physical Processes in the Lowest Layers of the Earth's Atmosphere, McGraw-Hill Book Company, New York, 1953.

8. D.A. Anderson, J.C. Tannehill and R.H. Pletcher, Computational Fluid Mechanics and Heat Transfer, Hemisphere Publishing Corporation, New York, 1984. 
Intentionally Left Blank 

Distribution List

MS1413 1114 T.A.Michalske

MS0841 9100 P.J.Hommert

MS0833 9103 J.H.Biffle

MS0828 9104 R.Thomas

MS0826 9111 W.Hermina

MS0834 9112 A.C.Ratzel

MS0835 9113 T.C.Bickel

MS0827 9114 R.Griffith

MS0825 9115 W.H.Rutledge

MS0833 9103 J.H.Biffle

MS0836 9116 C.W.Peterson

MS0443 9117/9118 H.S.Morgan

MS0437 9117/9118 C.Adams

MS0443 9117/9118 S.N.Burchett

MS0443 9117/9118 A.F.Fossum

MS0443 9117/9118 S.W.Key

MS0321 9200 W. Camp

MS9202 9202 R.J.Pryor

MS1111 9221 S.S.Dosanjh

MS1111 9221 C.C.Ober

MS1110 9222 R.C.Allen

MS1110 9222 D.E.Womble

MS1110 9223 D.Greenberg

MS1109 9224 A.L.Hale

MS1111 9225 G.Heffelfinger

MS0441 9226 R.Leland

MS0819 9231 J.M.McGlaun

MS0820 9232 P.Yarrington

MS0439 9234 D.R.Martinez

MS0439 9234 J.L.Dohner (10)

MS0439 9234 C.R.Dohrmann

MS0439 9234 T.Hinnerichs

MS0439 9234 B.J.Driessen (10)

MS0439 9234 D.J. Segalman

MS0557 9741 T.J.Baca

MS1003 9611 T.D. Robinett III

MS1003 9611 J.T. Feddema

MS1003 9611 B.L.Spletzer
MS1010 9622 M.E.Olson

MS1010 9622 G.R.Eisler

MS1008 9621 P.G.Xavier

MS1006 9671 J.E.Turner

MS1004 9623 K.S.Kwok

MS1004 9623 M.J.McDonald

MS1004 9623 E.J.Gottlieb

MS9018 8940-2 Central Tech. Files

MS0899 4916 Tech. Library (2)

MS0619 12690 Review \& Aproval Desk (2)

For DOE/OST I 